\title{
Visibility and Resorption: Are These Features Important?
}

\author{
Jafar Golzarian ${ }^{1}$
}

Received: 13 July 2020/Accepted: 2 August 2020/Published online: 19 November 2020

(C) Springer Science+Business Media, LLC, part of Springer Nature and the Cardiovascular and Interventional Radiological Society of Europe (CIRSE) 2020

Interventional Radiology is one of the most innovative specialties in medicine with new materials, techniques, and procedures frequently introduced to improve patient care and outcome. In recent years, the majority of these developments revolved around particle embolization. If in the late 1990s and early 2000s, we witnessed the emergence of Uterine Fibroid embolization, radioembolization, and improved understanding of basics of particle embolization; in the past decade new applications have surged exponentially. Procedures such as prostate artery embolization, embolization for joint pain in osteoarthritis, frozen shoulder or sports injuries, drug eluting TACE, and embolization for weight loss have emerged to mention a few. As a result, more researches were directed toward the understanding of factors affecting endpoints, development of new particles, microcatheters, improvement of targeting, and reduction of non-target embolization (NTE) [1].

In this issue of CVIR, Stechele et al. [2] have evaluated new resorbable and MRI visible particles in a rabbit kidney model. The particles were composed of polydioxanone impregnated with super-paramagnetic iron oxide (SPIO). The authors have been able to visualize the particles with MRI post embolization at different endpoints.

Ideally, imageable particles should be visible under fluoroscopy during the procedure and by other imaging modalities such as CT and MR in the follow-up. X-ray visibility is associated with technical challenges. To

Jafar Golzarian

golzarian@umn.edu

1 Radiology and Vascular Imaging, Amplatz Chair in Interventional Radiology, University of Minnesota, 420 Delaware Street S.E., B228-A Mayo Memorial Building, Minneapolis, MN 55455, USA become visible, particles need to be impregnated with higher atomic weight elements such as iodine, iron, barium, tungsten, and other heavy metals. The addition of these elements will change the physical characteristic of particles making them difficult to suspend in a solution and therefore their intravascular injection. MR visibility is technically less challenging. SPIO-based particles have been previously developed and tested in animals [3]. Although X-ray visibility is considered a very important milestone to prevent NTE and to improve lesion targeting, there are debates on whether MR visibility adds any value. SPIO impregnation of nanoparticles or cells such as stem cells has been subject to multiples research allowing tracking in the experimental and clinical settings [4]. At microparticles level, the detection in the follow-up MRI can help with the improvement of the technique of embolization to reduce NTE. A potential application could be the evaluation of particle embolization to the ovaries after uterine fibroid embolization and its relationship with fertility. MR visibility could allow a better understanding of clinical failure after a procedure such as prostate artery embolization. We can also learn much more about vascular collateral pathways, dynamics of embolization, and endpoints. Finally, if the detection can be associated with quantification, it could become a powerful tool in the management of cancer and other diseases.

The combination of visibility and resorbability is an interesting concept. In this study, even at 16 weeks, the SPIO signals were detected despite the resorption of the particles. The idea of resorption after the goal is achieved is gaining popularity within patients, physicians, and regulatory instances. The challenge in making resorbable microspheres larger than $300 \mu$ is mostly related to change of compressibility and injectability after sterilization with 
Gamma radiation. It is important to stress that revascularization is different from recanalization. A vessel can recanalize after embolization with permanent particles. Also, resorbable particles may result in permanent vessel occlusion even though the material is degraded. The duration of the resorption, the inflammatory profile of the materials, and the type of distal vascular bed are a few important factors affecting recanalization and revascularization $[1,5]$.

The ideal particle for embolization does not exist; however, some of the important characteristics of ideal particles are injectability, compressibility, ease of use, the potential to load any drugs, visibility during and after the procedure, and finally controlled resorption [1].

The evolution of particulate materials toward visibility and resorbability is essential in improving techniques of embolization, targeting, reducing NTE and long-term immune reaction, and optimizing outcome.

Funding None.

Compliance with Ethical Standards

Conflict of interest None.

Ethical Approval Not applicable.
Informed Consent Not applicable.

Consent for Publication The consent was obtained.

\section{References}

1. Golzarian J, Weng L. Particle embolization: factors affecting arterial distribution. J Vasc Interv Radiol. 2014;25(11):1773-4.

2. Stechele M, Wittgenstein H, Stolzenburg N, et al. Novel MRvisible, biodegradable microspheres for transcatheter arterial embolization: experimental study in a rabbit renal model. Cardiovasc Intervent Radiol. 2020. https://doi.org/10.1007/s00270-02002534-1.

3. Namur J, Chapot R, Pelage JP, et al. MR imaging detection of superparamagnetic iron oxide loaded tris-acryl embolization microspheres. J Vasc Interv Radiol. 2007;18(10):1287-95.

4. Frank JA, Miller BR, Arbab AS, Zywicke HA, et al. Clinically applicable labeling of mammalian and stem cells by combining superparamagnetic iron oxides and transfection agents. Radiology. 2003;228(2):480-7.

5. Weng L, Seelig D, Rostamzadeh P, et al. Calibrated bioresorbable microspheres as an embolic agent: an experimental study in a rabbit renal model. J Vasc Interv Radiol. 2015;26(12):1887-94.

Publisher's Note Springer Nature remains neutral with regard to jurisdictional claims in published maps and institutional affiliations. 